\title{
Developing New Q-meters for NMR Measurements of Polarized Solids
}

\author{
James Maxwell ${ }^{* \dagger}$ \\ Thomas Jefferson National Accelerator Facility \\ E-mail: jmaxwellajlab.org
}

\begin{abstract}
Enhanced polarization of target nuclei can be measured via the complex impedance of a resonant circuit coupled to the target while sweeping the circuit through the nuclear Larmor frequency, observing the absorption or emission of energy by the spins in the material. For decades, solid polarized targets at Jefferson Lab have relied upon continuous-wave Nuclear Magnetic Resonance polarization measurements using Liverpool Q-meter systems. These Liverpool Q-meters are no longer produced, and replacing the aging components is becoming increasingly difficult. The polarized target group at Bochum has produced replacement Q-meters in the Liverpool style using modern components, and we are following their example while researching improvemnts to the technique. We discuss our efforts to produce improved Q-meters for upcoming experiments utilizing solid polarized targets in Jefferson Lab's Hall B.
\end{abstract}

The 18th International Workshop on Polarized Sources, Targets, and Polarimetry, PSTP2019

23-27 September, 2019

Knoxville, Tennessee

\footnotetext{
*Speaker.

${ }^{\dagger}$ On behalf of the CLAS12 Polarized Target Collaboration.
} 


\section{Introduction}

Dynamic nuclear polarization (DNP) of solids allows the production of highly polarized targets which are a crucial piece of Jefferson Lab's polarized scattering program [1]. DNP takes advantage of the very high electron polarization $(P=\tanh (\mu B / k T)))$ at a practical temperature $(T \sim 1 \mathrm{~K})$ and magnetic field $(B \sim 5 T)$, to polarize nearby nuclei via flip-flop transitions in the electron-spinnucleus-spin system. These transitions are driven by microwave radiation of frequency close to the electron spin resonance frequency less or more the nuclear Larmor frequency ( $v_{\mathrm{esr}} \pm v_{\mathrm{nmr}}$ ).

Nuclear Magnetic Resonance (NMR) is used to measure the polarization achieved in a target sample. Using a coil of inductance $L_{0}$ near the sample and perpendicular to the polarizing field $B$, an induced field $B_{0}$ at the Larmor frequency $\omega_{0}$ drives spin flips, causing transitions of the Zeemansplitting energy levels, $\hbar \omega_{0}=g \mu B$. Depending on the average orientation of the spins to the field, these spins flips absorb or emit energy as they interact with the field generated by the inductor and its electrical circuit. The spin system's response to the induced RF field $B_{0}$ is its magnetic susceptibility, $\chi(\omega)$, and is a function of the RF frequency $\omega$. The magnetic susceptibility consists of a dispersive term $\chi^{\prime}(\omega)$ and an imaginary absorptive term $\chi^{\prime \prime}(\omega)$. This absorptive term can be integrated in frequency to give a proportional measure of the target polarization $P$ :

$$
\chi(\omega)=\chi^{\prime}(\omega)-i \chi^{\prime \prime}(\omega), \quad P=K \int_{0}^{\infty} \chi^{\prime \prime}(\omega) d \omega
$$

Here $K$ contains information on the target nucleus as well as the properties of the NMR system, and is typically determined empirically through measurements at thermal equilibrium, when the polarization can be determined from the known magnetic field and temperature via Boltzmann statistics. Since $\chi^{\prime \prime}(\omega)$ is non-zero only in a small range around the Larmor frequency, the integral only need be performed very close to center. The inductance of the embedded coil couples to the magnetic susceptibility, via a filling factor $\eta$,

$$
L_{C}(\omega)=L_{0}[1+4 \pi \eta \chi(\omega)]
$$

so that a measurement that isolates the real part of the complex impedance of the coil versus frequency will give a proportional measure of the polarization.

Jefferson Lab's solid polarized target experiments have traditionally utilized Liverpool Qmeters [2] to perform NMR measurements. The Q-meter observes a tank (LCR) circuit with the resistor and capacitor within the Q-meter connected via a transmission cable to the inductor embedded in the material at $1 \mathrm{~K}$, as shown in figure 1a. The tank circuit's adjustable capacitor allows the tuning of the circuit so that the resonant frequency, $\omega_{0}=1 / \sqrt{L C}$, can be set to the Larmor frequency of the nucleus of interest. The Q-meter's key component is a balanced ring modulator, a type of frequency mixer which compares the signal from the tank circuit to the reference excitation frequency, returning the real portion of the signal when the signal and reference match phase.

In the course of a measurement, the reference signal is swept through frequency, starting below the Larmor frequency and increased through it in discrete steps. For a well-tuned circuit, the Q-meter response results in an approximately symmetric background curve versus frequency, known as the Q-curve, which is due to reactive complex impedance below and above the resonant frequency. The sample polarization is determined by first removing this background Q-curve and 


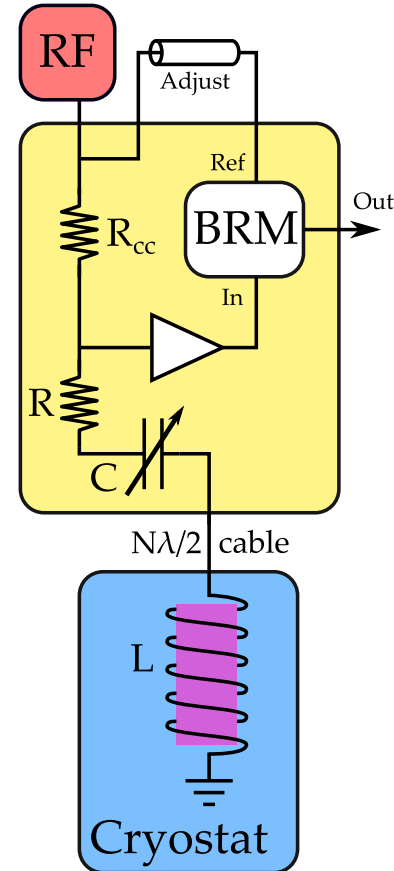

(a) Traditional Q-meter NMR

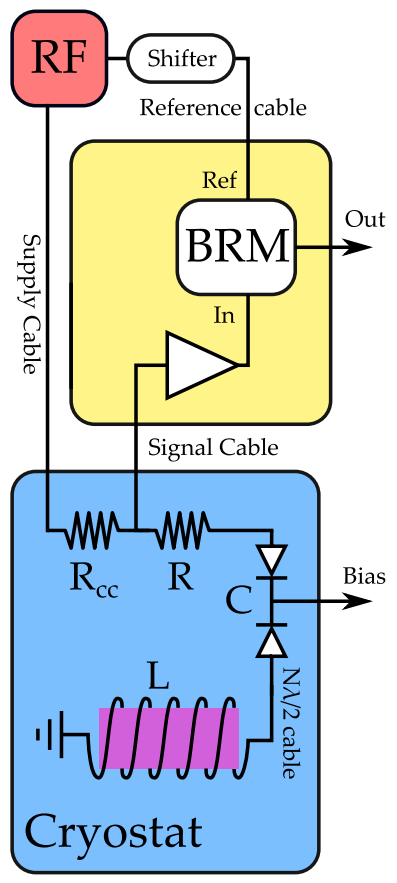

(b) "Cold" Q-meter NMR

Figure 1: Diagrams showing the traditional NMR layout, and the "cold" circuit NMR layout, which includes varactor-diode tuning capacitors and an electronic phase shifter.

integrating the remaining signal. The background Q-curve is measured by adjusting the magnetic field to be off resonance, and shifts in the background over time are removed through parameterized fits.

When performing calibrations using un-enhanced polarization of material at thermal equilibrium, and particularly when measuring deuteron signals, the signal size can approach the noise floor of the system. By moving the resistor and tuning capacitor into the cryostat in a "cold" circuit NMR layout, thermal noise can be reduced [3]. Because the tuning capacitor is no long accessible from outside the cryostat, variable capacitors, such as varactor diodes, can be used to remotely tune the resonant circuit [4], as shown in Figure 1b.

The lengths of the cable used to connect the components of the Q-meter system must be chosen carefully for an accurate measurement. In the traditional Q-meter layout, the long transmission line between the Q-meter (with internal resistor and tuning capacitor) and the coil allows a proper tune of the resonant circuit when the cable length is an integer multiple of half the wavelength of the RF $(N \lambda / 2)$, effectively removing the complex impedance of the transmission line at $\omega_{0}$. Because this length doesn't change as the frequency is swept, the cable contributes to the background Q-curve above and below $\omega_{0}$. The longer the $N \lambda / 2$ cable, the deeper the curve. In the cold circuit layout, the resistor, capacitor and inductor are together, removing this $N \lambda / 2$ transmission cable and its effect on the Q-curve. However, two transmission lines connect the cold circuit to the Q-meter, labeled as "signal" and "supply" in Figure 1b, and these cables contribute complex impedance to the measurement in different ways, as we discuss in section 2.1. 


\section{Development}

In preparation for a suite of polarized target experiments scheduled to run in Hall $\mathrm{B}$, we are working to improve our NMR techniques. The first priority to address is the aging of our Liverpool Q-meters, which have been in use since at least the 1990's. These units are quite robust, but they are no longer produced and include parts which are not replaceable. The Bochum target group has produced new Q-meters [5], following the original Liverpool design closely and utilizing an updated version of the same mixer, the Minicircuits SRA-1 balanced ring modulator [6]. To meet our needs, we have produced new Q-meter boards, expanding on the improvements they added.

Our second objective is to revisit cold circuit NMR techniques, which were used briefly for deuteron runs in JLab's Hall B polarized target [7] using static capacitors that did not allow remote tuning. We have added varactor diodes to allow remote tuning of the capacitance [8], and have been assessing their use not only for deuterons, but for protons as well. Tests of cold circuit NMR methods using in the CLAS12 polarized target refrigerator [9] have given unforeseen results. To date, our tests have focused on cold circuit NMR of proton signals at $5 \mathrm{~T}$ and $213 \mathrm{MHz}$, a very different regime than the $32.7 \mathrm{MHz}$ frequency of the deuteron at $5 \mathrm{~T}$. While the system performed as designed in other respects, we encountered polarization signals of the opposite magnitude, protruding out of the Q-curve rather than into it as expected. This effect was observed with our prototype Q-meter systems and with Liverpool Q-meters. Because no such effect was mentioned in Court et al [3] which discussed cold NMR at $32.7 \mathrm{MHz}$, we suspected that the higher frequency could contribute to the effect. To further investigate, we turned to calculations of the circuit behavior.

\subsection{Q-meter Calculation}

To anticipate the behavior of the Q-meter system in various experimental circumstances, we have developed computer code written in Python to calculate the Q-meter response. This work began from MathCAD code written by M. Houlden and G. Court, and was generalized to allow any length of transmission line in various circuit locations. The code contains equations describing the complex impedance of the resonant circuit and supporting elements, as prescribed by Kirchoff's laws, along with applications of the telegrapher's equations to account for long transmission lines. Notable are the terms required to account for the input impedance of the transmission lines:

$$
Z_{\text {in }}(\omega)=Z_{0} \frac{Z_{L}+Z_{0} \tanh (\gamma l)}{Z_{0}+Z_{L} \tanh (\gamma l)}
$$

for the load impedance $Z_{L}$. The characteristic impedance of the transmission line $Z_{0}$ and propagation constant $\gamma$ are expressed in terms of length $l$, resistance $R$, inductance $L$, capacitance $C$ and conductance $G$ of the line:

$$
Z_{0}(\omega)=\sqrt{\frac{R+i \omega L}{G+i \omega C}}, \quad \gamma(\omega)=\sqrt{(R+i \omega L)(G+i \omega C)} .
$$

The telegrapher's equations take into account the impedance mismatches which come with certain lengths of transmission cable, and they strongly effect the circuit response versus frequency.

Our code takes as input the tuning capacitance, phase shift applied to the reference input to the mixer, and the lengths of four possible transmission lines seen in Figure 1b: between the $C$ 
and $L$ of the resonant circuit (the traditional $N \lambda / 2$ line), between the RF generator and the constant current resistor $R_{\mathrm{Cc}}$ (the cold circuit supply line), between the resonant circuit signal and Q-meter amplifier (the cold circuit signal line), and/or between the RF generator and the mixer reference (the reference line). The output of the code are the "diode" signal (the signal magnitude) and the "phase" signal (the output of the mixer). It should be noted that this code does not simulate the mixer accurately. The balanced ring modulator used to down-mix the signal to the reference is treated as a perfect multiplier to implement the effect of the phase difference between the signal and reference. While this approximation is sufficient to understand most of the Q-meter's effects, we are working to include a more accurate formulation of the mixer's behavior.

In addition to simulating signals from known inputs, this code can also be used in reverse, as a function to fit baseline Q-meter signals and predict the inputs used to create them. Having a realistic fitting function allows more accurate fitting of baseline signal backgrounds. To extract the polarization signal, we typically fit a third order polynomial to the background Q-curve. Fits to a realistic Q-meter response can provide better representation of the Q-curve, reducing error on the polarization measurement due to to baseline subtraction.

To address the polarization signal reversal discussed in section 2, we used the code to look at varying cable lengths. The CLAS12 refrigerator is longer than the previous target, requiring at least $10 \lambda / 2$ cable at $213 \mathrm{MHz}$ to reach the target, and we suspected this extra cable could be involved in the effect. Figure 2 shows resonant circuit responses taken at the Q-meter's diode, where the magnitude of the complex impedance is displayed over a very large frequency band to illustrate the effect of different cables lengths. In Figure $2 \mathrm{a}$ the components are not separated by transmission lines, so a typical tank circuit response is seen, tuned with a minimum at $213 \mathrm{MHz}$. When a $10 \lambda / 2$ cable is introduced between the Q-meter and the coil of the inductor, strong nodes corresponding to impedance mismatches are seen in the frequency spectrum (Figure 2b). In the cold tank circuit layout, the supply line from the signal generator is impedance matched with a $50 \Omega$ resistor at the circuit, so that the effect of a $10 \lambda / 2$ cable is small, as seen in Figure 2c. However, the length of the signal cable (between the tank circuit and the Q-meter's amplifier) introduces nodes of a different sort, creating nearby local minima and maxima on top of the tank attenuation curve, as in Figure $2 d$.

These local minima and maxima due are the key to understanding the signal reversal effect. Varying the cable length or the tuning capacitance, and thereby shifting the central tune of Figure $2 \mathrm{~d}$ to the left or right, easily allows the centering of either a minima or maxima in the $800 \mathrm{kHz}$ window viewed in the Q-meter measurement. Looking from the Q-meter's narrow view, a phase change of 180 degrees flips both the Q-curve and the polarization signal, but choosing a maxima instead of a minima will flip the Q-curve but not the polarization signal. This means the signal reversal we observed was some combination of mismatched length, tuning capacitance and/or phase shift. To avoid signal distortion in the cold circuit layout, care must be taken in the selection of cable lengths (particularly the length of the signal cable), the tuning capacitance should not deviate greatly from that determined with short cables, and the overall DC offset of the signals should be observed. This effect is much exaggerated at $213 \mathrm{MHz}$ as the $\lambda / 2$ length is 6.7 times shorter than that at $32.7 \mathrm{MHz}$, where previous studies of cold circuits for deuterons have been performed. 


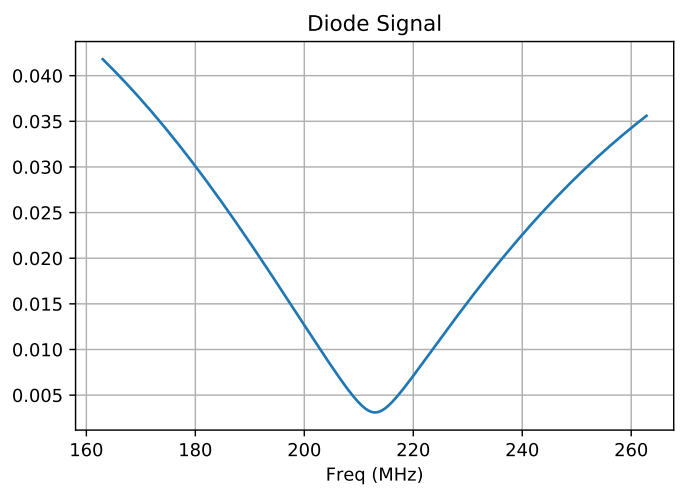

(a) Short cables

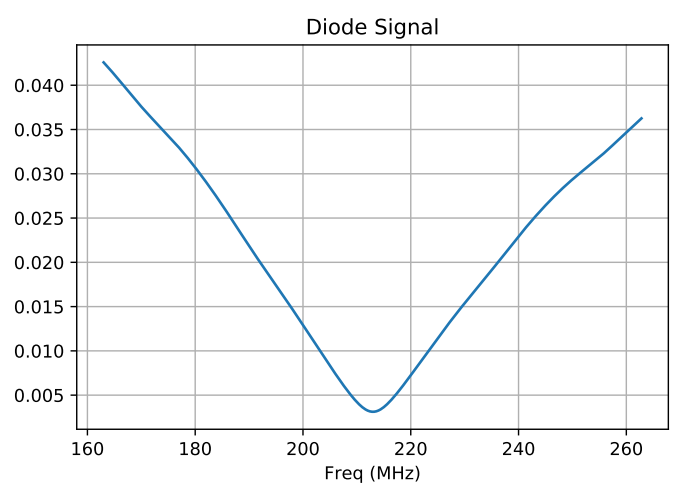

(c) $10 \lambda / 2$ supply cable

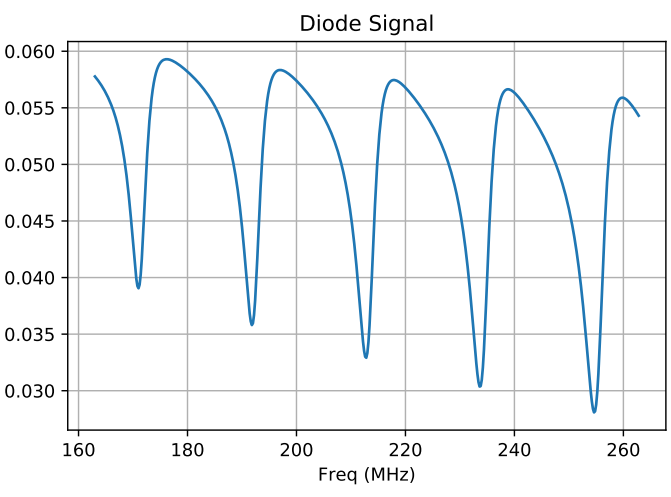

(b) $10 \lambda / 2$ coil cable

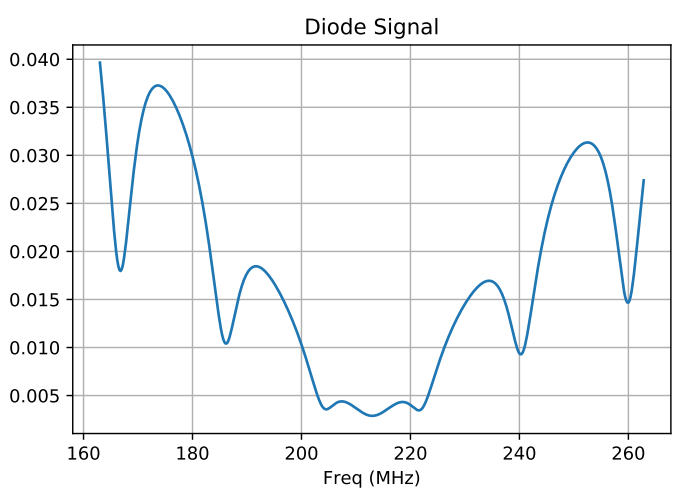

(d) $10 \lambda / 2$ signal cable

Figure 2: Calculated resonant circuit response over a wide frequency band, with different lengths of transmission cables. In Figures $2 \mathrm{~b}, 2 \mathrm{c}$ and $2 \mathrm{~d}$ one cable is lengthened, leaving the others short, to show its effect.

\subsection{Prototype Q-meter}

Our new Q-meter device follows the general design of the Liverpool Q-meter, with changes to take advantage of improvements in components and to improve the ergonomics of the measurements. The Liverpool design uses 5 circuits boards mounted side-by-side in a heavy brass chassis to provide temperature stability. These boards, listed by task, are: the tank circuit and signal splitter, the mixer, the phase amplifier, the diode amplifier, and the final amplifier. The boards and ground planes are generally separated to isolate large $\mathrm{AC}$ signals from the generator from smaller signals from the tank and DC signals after the rectifier diode and mixer. The mixer board receives the reference signal from the generator, splitting it to be sent through a length adjust phase shifter to the mixer and to the tank circuit on the first board.

Our design combines some of these tasks, while incorporating new boards for optional tasks. Our Q-meter includes 3 boards, as seen in Figure 3: a mixer board, phase amplification board, and diode/amplification board. The mixer board (Figure 3a) accepts the signal from the tank circuit and splits it to be sent to the mixer [6] and the separate diode board (Figure 3c). The tank circuit is moved outside the Q-meter, either to the interior of the cryostat in the cold circuit layout, or to immediately outside the Q-meter chassis, as previously done by the Bochum group. An optional 


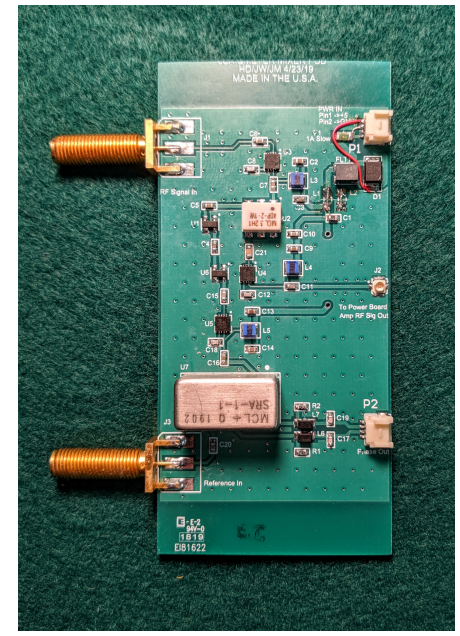

(a) Mixer board

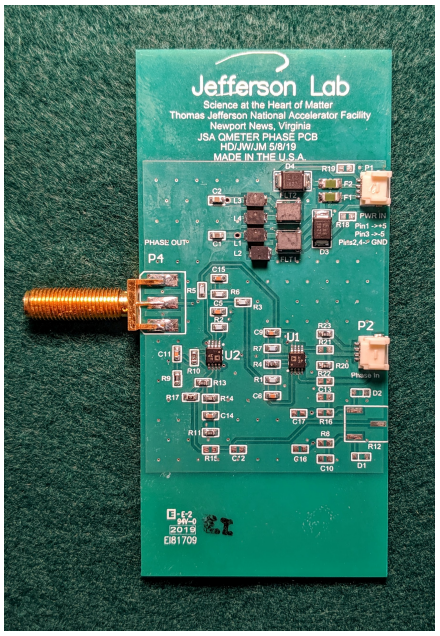

(b) Phase amplification board

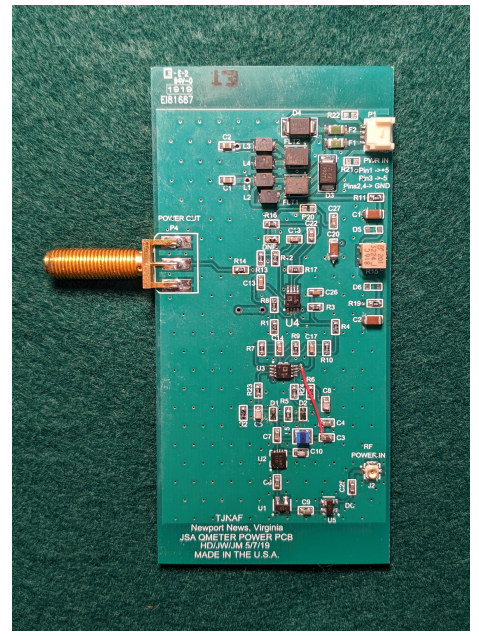

(c) Diode amplification board

Figure 3: Photographs of protoype Q-meter boards.

splitter/amplifier board prepares the signal to be sent to the tank and reference side of the mixer, and allows a electronic phase shifter chip to be used [10].

The most significant difference between our new design and those of Liverpool and Bochum is the amplifiers used. We have chosen Analog Devices amplifiers, simplifying the design to use one type of RF amplifier and one type of differential amplifier. The RF amplifiers (ADL5531) provide $20 \mathrm{~dB}$ of amplification and operate between 20 and $500 \mathrm{MHz}$, covering both $32.7 \mathrm{MHz}$ and $213 \mathrm{MHz}$ for deuterons and protons at $5 \mathrm{~T}$. The ADL5531 has a noise figure of $2.5 \mathrm{~dB}$, and replaces two Watkins-Johnson amps in the Liverpool: the A71 with a lower gain $(18 \mathrm{~dB})$ and comparable noise factor $(2.1 \mathrm{~dB})$ and the A54 with a higher gain $(27 \mathrm{~dB})$ and worse noise factor (4.5 dB). The differential amplifier package (ADA4896-2) provides two voltage feedback amplifier channels with low wideband noise $(1 \mathrm{nV} / \sqrt{\mathrm{Hz}})$, replacing GPD-403 $9 \mathrm{~dB}$ amplifiers with a $7.5 \mathrm{~dB}$ noise figure. These amplifiers have the added benefit of requiring only $5 \mathrm{~V}$ power supply, where the Liverpool required $24 \mathrm{~V}, \pm 15 \mathrm{~V}$ and $5 \mathrm{~V}$ for its chains of amplifiers.

The three Q-meter boards are sized to be slotted into off-the-shelf enclosures from Box Industries, stacked vertically and including shielding between each board while keeping the ground planes of each board isolated from the enclosure. We plan to include a heater in the enclosure to allow temperature stabilization of the assembly to reduce amplifier drifts over time.

\section{Outlook}

We are currently assessing the performance of our prototype Q-meter, and initial results are promising. We have measured enhanced proton signals in TEMPO-doped epoxy samples at $1 \mathrm{~K}$, and the signal-to-noise achieved appears to exceed measurements made using our Liverpool Qmeters, not including the Yale amplification card. Tests of the overall linearity, temperature dependence and a comparison with Yale gain card capabilities are being prepared. We have also begun the design of a bespoke ADC and DAC system to replace the National Instruments general purpose USB data acquisition boards we current use to record the output of the Q-meters. 
Our tests of the cold circuit NMR for $213 \mathrm{MHz}$ protons have encountered several difficulties. In addition to being more susceptible to transmission line effects than $32.7 \mathrm{MHz}$ deuterons, we have encountered strong resonances in the frequency spectrum that are hard to predict. These resonances show up as large peaks that appear in the frequency spectrum when viewed over a large band as the target is cooled below $20 \mathrm{~K}$. In the narrow-band view of the Q-meter, these peaks make tuning the circuit difficult as they are highly susceptible to thermal drifts inside the target cryostat. We are likely seeing parallel resonances to ground changing with the temperature dependence of the cable dielectric. The small tuning capacitance $(\sim 10 \mathrm{pF})$ required to tune at $213 \mathrm{MHz}$ exacerbates this effect. We are proceeding to tests of the cold circuit layout with varactor diode tuning capacitors at $32.7 \mathrm{MHz}$, where the lower thermal noise promised by this layout will be more valuable, and which we do not expect will suffer from the complications that have arisen at $213 \mathrm{MHz}$.

In addition to our work implementing a new Q-meter in the Liverpool style, we are investigating an "all digital" method to perform these measurements. This method would rely upon fast ADCs to directly perform measurements of the $213 \mathrm{MHz}$ signal rather than down mixing with a balanced ring modulator. We hope to have a proof-of-principle prototype to begin testing this technique late in 2020.

Hall B's polarized target experiments are expected to commence in 2022, when our new Qmeter system should be ready to debut alongside the new CLAS12 horizontal DNP refrigerator [9]. We do not anticipate that our existing Q-meter prototype boards will require significant alteration once testing is complete, as they have operated well for several cool-downs.

\section{Acknowledgements}

We gratefully acknowledge the contributions of the members of the CLAS12 polarized target collaboration, especially the C. Keith and JLab Target Group, H. Dong and the JLab Electronics Group, J. Pierce of ORNL, and V. Lagerquist of ODU. This material is based on work supported by the U.S. Department of Energy, Office of Science, Office of Nuclear Physics under contract DE-AC05-06OR23177.

\section{References}

[1] D. G. Crabb and W. Meyer, Solid polarized targets for nuclear and particle physics experiments, Annual Review of Nuclear and Particle Science 47 (1997) 67.

[2] G. Court, D. Gifford, P. Harrison, W. Heyes and M. Houlden, A high precision q-meter for the measurement of proton polarization in polarised targets, Nuclear Instruments and Methods in Physics Research Section A 324 (1993) 433.

[3] G. Court, M. Houlden, S. Bultmann, D. Crabb, D. Day, Y. Prok et al., High precision measurement of the polarization in solid state polarized targets using $\mathrm{nmr}$, Nuclear Instruments and Methods in Physics Research Section A 527 (2004) 253.

[4] E. J. Veenendaal, R. Hulstman and H. B. Brom, A frequency-modulated q-meter for very low-temperature nmr experiments, Journal of Physics E: Scientific Instruments 16 (1983) 649.

[5] J. Herick, Development of a new Q-meter module, in Proceedings, 16th International Workshop on Polarized Sources, Targets, and Polarimetry (PSTP 2015): Bochum, Germany, September 14-18, 2015, vol. PSTP2015, p. 011, 2016, https://pos.sissa.it/243/011/pdf. 
[6] MiniCircuits, "Double balanced mixer, https://www.minicircuits.com/pdfs/SRA-1-1+.pdf."

[7] C. Keith, M. Anghinolfi, M. Battaglieri, P. Bosted, D. Branford, S. Bultmann et al., A polarized target for the clas detector, Nuclear Instruments and Methods in Physics Research Section A: Accelerators, Spectrometers, Detectors and Associated Equipment 501 (2003) 327.

[8] J. D. Maxwell, NMR Measurements for Solid Polarized Targets at Jefferson Lab, PoS SPIN2018 (2019) 102.

[9] J. Brock, 1 K Refrigerator for the CLAS12 Polarized Target: Design, Construction, and First Results, in These Proceedings, 2020.

[10] MiniCircuits, "Surface mount phase shifter, https://www.minicircuits.com/pdfs/SPHSA-251+.pdf." 